\title{
Total cross sections and $\rho$ at high energy
}

\author{
O. V. Selyugin \\ BLTPh, JINR, Dubna, Russia
}

\begin{abstract}
Analysis of the new experimental data obtained by the TOTEM Collaboration at LHC at $\sqrt{s}=7 \mathrm{TeV}$ at small momentum transfer is presented. The impact of the different assumptions on the extraction of the parameters of the elastic scattering amplitude, especially on the size of the total cross sections, is examined. It is shown that the contribution of the Coulomb amplitude and Coulomb-hadron interference term should be taken into account in the analysis of the existing experimental data at small momentum transfer. Our new method of extracting the real part of the hadron scattering amplitude from experimental data shows the inconsistency of the size of $\rho=0.14$ to the parameters of the imaginary part of the hadron scattering amplitude obtained by the TOTEM Collaboration. The analysis of the data is compared with the similar analysis in the Regge approach for the hadron scattering amplitude.
\end{abstract}

Keywords: Hadron, total cross sections, elastic scattering, high energies

\section{Introduction}

The determination of the parameters of the elastic scattering amplitude, such as $\sigma_{\text {tot }}$ - total cross sections, $\rho(s, t)$ - ratio of the real to imaginary part of the scattering amplitude, $B(s, t)$ - slopes of the imaginary and real parts of the scattering amplitude is one of the important tasks of experimental researches at the LHC. The properties of the elastic scattering amplitude at small angles are tightly connected, on the one hand, with the first principles of the theory of strong interactions and, on the other hand, with the nonperturbative properties of the hadron interaction. The knowledge of the size of the total, elastic and inelastic cross sections is important for other experimental researches at LHC. As part of the SMC Collaboration of the 
LHC CERN the TOTEM Collaboration (TOTal and Elastic scattering crosssection Measurement) is one of the special purpose experiments to obtain the new information about the elastic hadron scattering at LHC energies at wide momentum transfer [1]. Under various beam and background conditions, the differential elastic and elastic, inelastic total proton proton cross sections have been measured. Now they published [2] the data on the differential elastic cross sections at $\sqrt{s}=7 \mathrm{TeV}$ at the sufficiently large momentum transfer $\left(0.3 \leq|t| \leq 2.5 \mathrm{GeV}^{2}\right.$ and small $5 \cdot 10^{-3} \leq|t| \leq 0.4 \mathrm{GeV}^{2}$ using the size of the Luminocity obtained by the SMC Collaboration. They obtained that the slope $B=19.9 \pm 0.3 \mathrm{GeV}^{-2}$ under the assumption that the differential cross section in the region of $5 \cdot 10^{-3} \leq|t| \leq 0.2 \mathrm{GeV}^{2}$ can be described by exponent with the slope independent of $t$. Extrapolation of the differential cross sections at $t=0$ and the optical theorem gave the size of the total cross sections $\sigma_{\text {tot }}=98.7 \mathrm{mb}$. They used the size of $\rho=0.141 \pm 0.007$ (ratio of the real to imaginary part of the elastic scattering amplitude) from the analysis carried out by the COMPETE (Computerised Models, Parameter Evaluation for Theory and Experiment) Collaboration [3] as preferred-model extrapolation. The COMPETE Collaboration considered several hundreds of possible parametrization for $p p, \bar{p} p, \pi^{ \pm} p, K^{ \pm} p, \gamma p$ and $\gamma \gamma$, based on simple, double or triple poles, and kept only those which had a global $\chi^{2} /$ point smaller than 1, for $\sqrt{s} \geq 5 \mathrm{GeV}$. From these, one can predict $\rho$ and $\sigma_{\text {tot }}$ at the LHC, and estimate the error due to the extrapolation. The first results obtained by the TOTEM collaboration [2, 4] on the differential cross sections are in disfavor with practically all theoretical model predictions [2, 5]. Now the models are reconstructed to obtain the coincidence with the new experimental results.

The number of elastic events is related to the total hadronic cross section through

$$
\begin{aligned}
\frac{d N}{d t}=\quad & \mathcal{L}\left[\frac{4 \pi \alpha^{2}}{|t|^{2}} G^{4}(t)-\frac{2 \alpha\left(\rho(s, t)+\phi_{C N}(s, t)\right) \sigma_{t o t} G^{2}(t) e^{-\frac{B(s, t)|t|}{2}}}{|t|}\right. \\
& \left.+\frac{\sigma_{\text {tot }}^{2}\left(1+\rho(s, t)^{2}\right) e^{-B(s, t)|t|}}{16 \pi}\right]
\end{aligned}
$$

where the three terms are due to the Coulomb scattering, Coulomb-hadron interference and hadronic interactions; $\mathcal{L}$ is the integrated luminosity, $\alpha$ is the electromagnetic coupling constant, $\phi_{C N}(s, t)$ is the Coulomb-hadron phase, 
and $G(t)$ is the electromagnetic form factor given by

$$
G(t)=\frac{4 m_{p}^{2}-\mu t}{4 m_{p}^{2}-t} \frac{\Lambda^{2}}{(\Lambda-t)^{2}} .
$$

with $m_{p}$ being the proton mass, $\Lambda=0.71 \mathrm{GeV}^{2}$ and $\mu=2.79$.

When we extract the parameters of the scattering amplitude from the experimental data, we need to use some theoretical assumption and approximations. For example, in [6, 7] it was shown that the saturation regime, which can occur at the LHC energies, changes the behavior of the slope of the differential cross sections at small momentum transfer. As a result, the differential cross section cannot be described by a simple exponential form with the constant slope. Note, in (1) the assumption of the equality of the slopes of the imaginary and real parts $\left(B_{R e}(s, t)=B_{I m}(s, t)\right)$ was used.

A remarkable example was obtained from the analysis of the experimental data at $S p \bar{p} S$. In the proton-antiproton scattering at $\sqrt{s}=540 \mathrm{GeV}$ there are two different measures of the size of $\rho$ : the UA4 Collaboration $(\rho=0.24)$ and the UA4/2 Collaboration $(\rho=0.139)$. However, more careful analysis gave $\rho=0.19$ for the data of UA4 [8] and $\rho=0.16$ for the data UA4/2 [9] ). Hence, the contradictions between the experimental data practically disappeared. In [9], it was shown that $\chi^{2}$ in the fitting of the experimental data decreases by $10 \%$ if we use the slope in a more complicated form $B(t)=B_{0} t+C \sqrt{t_{0}-t}$. It changes the form of the differential cross section at very small momentum transfer.

In the present paper, we made some analysis of the new experimental data obtained by the TOTEM Collaboration at $\sqrt{s}=7 \mathrm{TeV}$ at small momentum transfer. We used the phenomenological model with exponential behavior of the scattering amplitude. It is the simplest model with many different assumptions. Some of them will be discussed in this paper. In this model, we need to take $\rho(s, t)$ as a free parameter or, as made by the TOTEM Collaboration, from the previous fit of the COMPETE Collaboration. Then we take the simple Regge approaches, where the real part of the scattering amplitude is determined by the intercept $\alpha(0)-1$ of the Regge trajectory.

The differential cross sections of the nucleon-nucleon elastic scattering can be written as the sum of different helicity amplitudes:

$$
\frac{d \sigma}{d t}=\frac{2 \pi}{s^{2}}\left(\left|\Phi_{1}\right|^{2}+\left|\Phi_{2}\right|^{2}+\left|\Phi_{3}\right|^{2}+\left|\Phi_{4}\right|^{2}+4\left|\Phi_{5}\right|^{2}\right) .
$$


The total helicity amplitudes can be written as a sum of nuclear $\Phi_{i}^{h}(s, t)$ and electromagnetic $\Phi_{i}^{e}(s, t)$ amplitudes $\Phi_{i}(s, t)=F_{i}^{h}(s, t)+F_{i}^{\mathrm{em}}(s, t) e^{\varphi(s, t)}$, where $\varphi(s, t)$ is the interference phase factor between the electromagnetic and strong interactions [10, 11, 12]. We assume, as usual, that at high energies and small angles the one-flip and double-flip hadron amplitudes are small with respect to the spin-nonflip ones and that the hadron contributions to $\Phi_{1}$ and $\Phi_{3}$ are the same.

The electromagnetic amplitude can be calculated in the framework of QED. In the high energy approximation, it can be obtained [13] for the spinnon-flip amplitudes:

$$
F_{1}^{e m}(t)=\alpha f_{1}^{2}(t) \frac{s-2 m^{2}}{t} ; \quad F_{3}^{e m}(t)=F_{1}^{e m}
$$

and for the spin-flip amplitudes:

$$
\begin{array}{r}
F_{2}^{e m}(t)=\alpha \frac{f_{2}^{2}(t)}{4 m^{2}} s ; \quad F_{4}^{e m}(t)=-F_{2}^{e m}(t), \\
F_{5}^{e m}(t)=\alpha \frac{s}{2 m \sqrt{|t|}} f_{1}(t) f_{2}(t),
\end{array}
$$

where the form factors are:

$$
f_{1}(t)=\frac{4 m_{p}^{2}-\mu t}{4 m_{p}^{2}-t} G_{d}(t) ; \quad f_{2}(t)=\frac{4 m_{p}^{2}(\mu-1)}{4 m_{p}^{2}-t} G_{d}(t)
$$

where $G_{d}(t)$ has the conventual dipole form

$$
G_{d}(t)=1 /(1-t / 0.71)^{2}
$$

\section{Experiment and analysis}

The hadron spin non-flip amplitude was chosen in the form taking into account the possible non-exponential form (using the nearest $t$-channel singularity [14, 15, 5])

$$
F(s, t)=(i+\rho) \frac{\sigma_{t o t}}{4 k \pi} e^{\left.\left[B / 2 t+C / 2\left(\sqrt{4 m_{\pi}^{2}-t}-2 m_{\pi}\right)\right]\right)}
$$

where $m_{\pi}=0.139 \mathrm{GeV}$ is pion mass and $k=0.38938 \mathrm{mb} \mathrm{GeV}^{-2}, t=-q^{2}$, and $C \mathrm{GeV}^{-1}$ is some coefficient which is determined by some additional 
part of the slope. In most part we will examine the set of the TOTEM data at small $t$ with $N=47$ points and $-t_{\max }=0.112 \mathrm{GeV}^{2}$ and some shortest interval with $N=40$ points and $-t_{\max }=0.097 \mathrm{GeV}^{2}$. The whole set $\left(N=86\right.$ points and $-t_{\max }=0.3 \mathrm{GeV}^{2}$ we will examined only as an example. This interval of momentum transfer is large and the imaginary part of the scattering amplitude may have some complicated form. In all our calculations we used only statistical errors of the experimental data.

\begin{tabular}{c|c|c|c|c|c|c}
\hline $\mathrm{i}$ & $N$ & $\sum_{i=1}^{N} \chi_{i}^{2}$ & $\rho$ & $B$ & $C$ & $\sigma_{\text {tot }}, m b$ \\
\hline & & & & & & \\
1 & 86 & 287. & $0.14 \mathrm{fix}$ & 20. & $0 . f i x$ & $98.87 \pm 0.1$ \\
2 & 86 & 287 & $0.05 \mathrm{fix}$ & 20. & $0 . f i x$ & $99.7 \pm 0.1$ \\
3 & 86 & 287 & $0.146 \pm 0.3$ & 20. & $0 . f i x$ & $98.8 \pm 0.4$ \\
4 & 86 & 220.5 & 0.14 fix & 21.7 & $-1.4 \pm 0.2$ & $97.9 \pm 0.2$ \\
5 & 86 & 220. & $0.05 \pm 0.4$ & 21.8 & $-1.4 \pm 0.2$ & $98.76 \pm 4$. \\
\hline
\end{tabular}

Table 1: The basic parameters of the model are determined by fitting experimental data without the electromagnetic contributions and with free $\sigma_{t o t}$.

\begin{tabular}{c|c|c|c|c|c|c}
\hline $\mathrm{i}$ & $N$ & $\sum_{i=1}^{N} \chi_{i}^{2}$ & $\rho$ & $B$ & $C$ & $\sigma_{\text {tot }}, \mathrm{mb}$ \\
\hline & & & & & & \\
1 & 47 & 64.96 & $0.176 \pm 0.2$ & 19.9 & $0 . f i x$ & $98.05 \pm 1.7$ \\
2 & 47 & 64.96 & $0.15 \mathrm{f}$ ix & 19.9 & $0 . f i x$ & $98.47 \pm 0.1$ \\
3 & 47 & 64.96 & $0.14 \mathrm{fix}$ & 19.9 & $0 . f i x$ & $98.6 \pm 0.1$ \\
4 & 47 & 64.96 & $0.1 \mathrm{fix}$ & 19.9 & $0 . f i x$ & $99.1 \pm 0.1$ \\
5 & 47 & 64.96 & $0.05 \mathrm{fix}$ & 19.9 & $0 . f i x$ & $99.44 \pm 0.1$ \\
6 & 47 & 64.96 & $0.0 \mathrm{fix}$ & 19.9 & $0 . f i x$ & $99.57 \pm 0.1$ \\
7 & 47 & 64.96 & $-0.05 \mathrm{fix}$ & 19.9 & $0 . f i x$ & $99.44 \pm 0.1$ \\
8 & 47 & 61.09 & $0.14 \mathrm{fix}$ & 18.5 & $1.05 \pm 0.54$ & $98.99 \pm 0.2$ \\
9 & 47 & 61.09 & $0.1 \mathrm{fix}$ & 18.5 & $1.06 \pm 0.54$ & $99.47 \pm 0.2$ \\
10 & 47 & 61.09 & $0.0 \mathrm{fix}$ & 18.5 & $1.07 \pm 0.54$ & $99.97 \pm 0.2$ \\
11 & 47 & 60.08 & $-0.03 \pm 0.1$ & 18.4 & $1.07 \pm 0.54$ & $99.94 \pm 0.4$ \\
\hline
\end{tabular}

Table 2: The basic parameters of the model are determined by fitting experimental data without the electromagnetic contributions. 
First, let us make the fit of the differential cross sections with the hadronic amplitude in form (8) and not take into account the electromagnetic interactions. The result of the fit for $N=86$ is presented in Table 1 . The $\sum_{i=1}^{N} \chi_{i}^{2}$ is large for all variants.

Hence, let us examine the interval of $t$ like the interval of the experimental data of the UA4/2 Collaborations. It includes the $N=47$ experimental points. The result of the fit is presented in Table 2. The first row shows

\begin{tabular}{c|c|c|c|c|c}
\hline$N$ & $\sum_{i=1}^{N} \chi_{i}^{2}$ & $\rho$ & $B$ & $C$ & $\sigma_{\text {tot }}, m b$ \\
\hline & & & & & \\
86 & 281. & 0.14 fixed & 20. & 0.fix & $99.4 \pm 0.1$ \\
86 & 281. & 0.1 fix & 20. & 0.fix & $99.7 \pm 0.1$ \\
86 & 288. & 0. fix & 20. & 0.fix & $99.8 \pm 0.1$ \\
86 & 245. & 0.14 fix & 21.3 & $-1.03 \pm 0.2$ & $98.6 \pm 0.2$ \\
86 & 215. & 0.0 fix & 21.8 & $-1.2 \pm 0.2$ & $98.7 \pm 0.2$ \\
86 & 175. & $-0.41 \pm 0.1$ & 23.2 & $-2.77 \pm 0.2$ & $89.1 \pm 3$. \\
\hline
\end{tabular}

Table 3: The basic parameters of the model are determined by fitting experimental data with $N=86$.

\begin{tabular}{c|c|c|c|c|c}
\hline$N$ & $\sum_{i=1}^{N} \chi_{i}^{2}$ & $\rho$ & $B$ & $C$ & $\sigma_{\text {tot }}, m b$ \\
\hline & & & & & \\
47 & 87.1 & 0.2 fixed & 20.1 & 0. fix & $98.5 \pm 0.1$ \\
47 & 77.1 & 0.14 fixed & 20. & 0. fix & $99.2 \pm 0.1$ \\
47 & 71.6 & 0.1 fix & 20 & 0. fix & $99.5 \pm 0.1$ \\
47 & 61.1 & $-0.07 \pm 0.05$ & 19.8 & 0. fix & $98.93 \pm 0.8$ \\
47 & 61.2 & 0.1 fix & 17.7 & $1.66 \pm 0.54$ & $100.1 \pm 0.2$ \\
47 & 60.6 & 0.0 fix & 18.8 & $0.82 \pm 0.54$ & $99.8 \pm 0.2$ \\
47 & 60.6 & $0.01 \pm 0.1$ & 18.9 & $0.74 \pm 0.8$ & $99.7 \pm 0.8$ \\
\hline
\end{tabular}

Table 4: The basic parameters of the model are determined by fitting experimental data with $N=47$.

the calculation with variation of three parameters: $\rho$, slope $-B$ and $\sigma_{\text {tot }}$. The minimum in $\sum_{i=1}^{N} \chi_{i}^{2}$ in the fitting procedure is very wide which leads to the large errors of the determined sizes of $\rho$ and $\sigma_{\text {tot }}$. Let us fix the size 
of $\rho$. The next 6 rows (2-7) present the fit with a different fixed size of $\rho$ and with $C=0$. We see that $\sum_{i=1}^{N} \chi_{i}^{2}$ is independent of the size of $\rho$ and $\sigma_{\text {tot }}$ has a small change. The size of $\sigma_{\text {tot }}$ is slightly above the data obtained by the TOTEM Collaboration [4]. In the next 3 rows (8-10) the fit includes an additional part of the slope which is proportional to the coefficient $C$. The $\chi^{2}$ decreases slightly, which reflects the presence of the additional free parameter, but again the size of $\rho$ is badly determined. The size of $\sigma_{t o t}$ increases slightly, but the errors of $\sigma_{t o t}$ increase essentially. The last row (11) presents the attempt to fit with all free parameters. In this case, we obtain a small value of $\rho$ and large $\sigma_{\text {tot }}$.

Hence, we can conclude that in neglecting the electromagnetic contribution the size of $\rho$ practically does not impact the determination of $\sigma_{\text {tot }}$ in this region of momentum transfer. Such assumption was made by the TOTEM Collaboration in the fitting procedure.

Now let us make the same fit but include the electromagnetic part of the elastic scattering amplitude. Of course, it is sufficiently small in this range of the momentum transfer; however, it leads to visible results. The fits for the number of the experimental points 86 are shown in Table 3. The size of $\sum_{i=1}^{N} \chi_{i}^{2}$ is large except for the last row. But in this case we obtain the unusual sizes of $\rho$ and $\sigma_{\text {tot }}$.

\begin{tabular}{c|c|c|c|c|c}
\hline$N$ & $\sum_{i=1}^{N} \chi_{i}^{2}$ & $\rho$ & $B$ & $C$ & $\sigma_{\text {tot }}, m b$ \\
\hline & & & & & \\
40 & 78.8 & 0.2 fix & 20.1 & 0.fix & $98.6 \pm 0.12$ \\
40 & 70.4 & 0.14 fix & 20. & 0.fix & 99.29 \\
40 & 65.8 & 0.1 fix & 20 & 0.fix & $99.55 \pm 0.12$ \\
40 & 56.6 & $-0.076 \pm 0.06$ & 19.8 & 0. fix & $98.83 \pm 0.12$ \\
40 & 54.7 & 0.1 fix & 16.3 & $2.63 \pm 0.8$ & $100.3 \pm 0.27$ \\
40 & 54.9 & 0.0 fix & 17.8 & $1.47 \pm 0.8$ & $99.96 \pm 0.26$ \\
40 & 54.6 & $0.06 \pm 0.01$ & 16.9 & $2.17 \pm 1.4$ & $100.3 \pm 0.3$ \\
\hline
\end{tabular}

Table 5: The basic parameters of the model are determined by fitting experimental data with $N=40$.

In the case with $N=47$ (Table 4 ), $\sum_{i=1}^{N} \chi_{i}^{2}$ is larger than in Table 2 for the first three rows, but for other cases it is almost the same. The first 4 rows present the fit without the additional part of the slope. In this case, 


\begin{tabular}{c|c|c|c|c|c}
\hline$N$ & $\sum_{i=1}^{N} \chi_{i}^{2}$ & $\rho$ & $B$ & $C$ & $\sigma_{\text {tot }}, m b$ \\
\hline & & & & & \\
47 & 134.5 & 0.14 fixed & 19.8 & 0. - fixed & 98.4 \\
47 & 174.7 & 0.1 fix & 19.7 & 0.fix & 98.4 \\
47 & 88.1 & $0.203 \pm 0.01$ & 20.1 & 0.fixed & 98.4 \\
47 & 105.3 & 0.14 fixed & 22.9 & $-2.3 \pm 0.3$ fixed & 98.4 \\
47 & 61.4 & $-0.105 \pm 0.02$ & 20. & $-0.14 \pm 0.4$ & 98.4 \\
\hline
\end{tabular}

Table 6: The basic parameters of the model are determined by fitting experimental data with fixed $\sigma_{t o t}$.

\begin{tabular}{c|c|c|c|c|c}
\hline$N$ & $\sum_{i=1}^{N} \chi_{i}^{2}$ & $\rho$ & $B$ & $C$ & $\sigma_{\text {tot }}, m b$ \\
\hline & & & & & \\
40 & 88.17 & $0.203 \pm 0.007$ & 20.1 & $0 .-$ fixed & 98.4 \\
40 & 88.2 & 0.2 fixed & 20. & $0 .-$ fixed & 98.4 \\
40 & 125.6 & 0.14 fixed & 19.7 & $0 .-$ fixed & 98.4 \\
40 & 157.7 & 0.1 fix & 19.6 & $0 . f i x$ & 98.4 \\
40 & 102.4 & $0.14 f i x$ & 22.4 & $-1.75 \pm 0.4$ & 98.4 \\
40 & 56.9 & $-0.106 \pm 0.01$ & 19.8 & $0 . f i x$ & 98.4 \\
40 & 56.8 & $-0.11 \pm 0.02$ & 19.7 & $0.1 \pm 0.7$ & 98.4 \\
\hline
\end{tabular}

Table 7: The basic parameters of the model are determined by fitting experimental data with fixed $\sigma_{t o t}$.

the influence of the size of $\rho$ is visible and we can make the fit taking $\rho$ as free parameters (the row 4). We obtained the negative size of $\rho$ at minimum $\sum_{i=1}^{N} \chi_{i}^{2}$. It is essentially far away from the TOTEM Collaboration analysis and the predictions of the COMPETE Collaboration. However, the size of $\sigma_{\text {tot }}$ is the same as in Table 2 in the region of errors. If we take the additional part of the slope (the rows 5-7 of Table 4), the error of $\rho$ increases and its size is badly determined. But the size of the coefficient $C$ is determined well especially with the fixed size of $\rho$. In this case, the size of $\sigma_{t o t}$ increases.

If a slightly less interval of momentum transfer (40 points with $-t \leq$ $0.097 \mathrm{GeV}^{2}$ ) is taken, the whole picture will be the same (see Table 5), but the size of the coefficient $C$ is determined better and its size reaches $2.6 \pm 0.8 \mathrm{GeV}^{-1}$. Remarkable, the size of $\sigma_{\text {tot }}$ is practically the same as in 
the previous case.

We can check some parts of our assumptions included in the fit procedure. If we know the parameters of the imaginary part of the hadron scattering amplitude, then it is possible to calculate the real part [8] using the experimental data on the differential cross sections and taken into account that for the proton-proton interaction $\operatorname{ReF}_{C}(t)<0$.

$$
\begin{aligned}
\operatorname{ReF}^{h}\left(t_{i}\right) & =-\operatorname{Re}_{C}\left(t_{i}\right) \\
& \pm \quad\left[\left[\left.\frac{d \sigma}{d t_{i}}\right|_{\text {exp. }}-k \pi *\left(I m F_{C}\left(t_{i}\right)+I m F_{h}\right)^{2}\left(t_{i}\right)\right] /(k \pi)\right]^{1 / 2}
\end{aligned}
$$

here the sign $(+)$ in the case $R e F_{h} \geq\left|R e F_{C}\right|$ and sign (-) in the case when $\left|R e F_{C}\right|>R e F_{h}$. Let us take the imaginary part of the hadron scattering amplitude in the simple exponential form with the parameters obtained by the TOTEM Collaboration

$$
\operatorname{Im} F^{h}(t)=\sigma_{t o t} /(4 k \pi) e^{B t / 2}
$$

with $\sigma_{t o t}=98.6 \mathrm{mb}$ and $B=19.9 \mathrm{GeV}^{-2}$.

The obtained results are shown in Fig.1 by the triangles (when we neglect the Coulomb amplitude) and by the squares (with taking into account the Coulomb amplitude. If the square of the sum of the imaginary parts of the Coulomb and hadron amplitudes exceeds the experimental data, then the value under the square root will be negative and the imaginary part of the calculations appears. With the imaginary part of the amplitude calculated with the parameters of the TOTEM Collaboration we obtain the same situation for many experimental points at larger momentum transfer in both cases (with and without the electromagnetic contributions). In Fig.1, such an imaginary part (multiplied by $-i$ ) is presented by the empty triangles and squares. Compare such results with the real part which is assumed in the fit procedure of the TOTEM Collaboration

$$
R e F^{h}(t)=\rho \sigma_{t o t} /(4 k \pi) e^{B t / 2},
$$

with $\rho=0.14$ which is obtained by the COMPETE analysis and used by the TOTEM Collaboration. In Fig.1, this real part of the hadron amplitude is presented by the long -dashed line. Obviously, the line coincides in most part, except the regions of the very small and large $t$, with the case when we take into account the Coulomb amplitude. We also see that at large 
momentum transfer the contribution of the imaginary part of the scattering amplitude exceeds the experimental data. This effect shows either some problem with the normalization, or the non-exponential form of the hadron scattering amplitude.

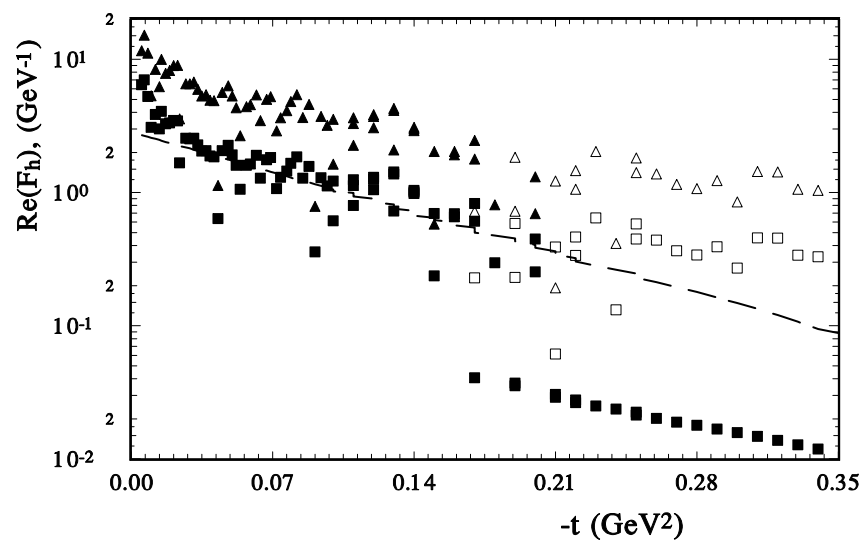

Figure 1: Real part of the hadronic amplitude calculated by eq.(9)(triangles and squared without and with $F_{c}$; [solid and empty represent real and imaginary parts of eq.(9) see text]; long dashed line - the calculations by eq.(111)).
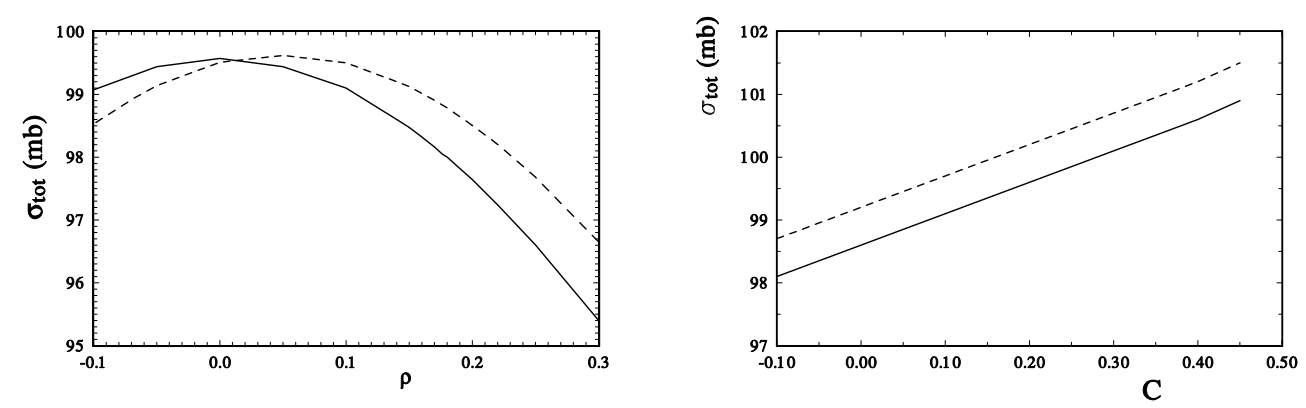

Figure 2: Size of $\sigma_{\text {tot }}$ (left) as a function of $\rho$ and (right) $C$ (hard line - without electromagnetic interaction and dashed line with electromagnetic interaction).

Now let us make the fit with the fixed size of $\sigma_{\text {tot }}=98.4 \mathrm{mb}$ (see Table 6 for a wide interval of $t$; and Table 7 , for a narrow interval of $t$ ). In this case, if we take $C=0, \chi^{2}$ increases essentially. The size of $\rho$ is determined well with the fixed $C$ and with the free $C$. Again, as in the first case, we obtain the negative size of $\rho$ near value -0.1 for both the cases and in the cases of the short and long intervals of $t$. The size of the coefficient $C$ is determined 
badly in both the cases and has a negative sign, but its size is less than the size of $C$, presented in Tables 2-5.

In Fig. 2, the dependence of the size of $\sigma_{t o t}$ as a function of $\rho$ (left picture) and as a function of slope $C$ (right picture) in the case without and with the contributions of the Coulomb and Coulomb-hadron interference terms is shown. The inclusion of the Coulomb dependence terms leads to an increase of $\sigma_{\text {tot }}$ at large $\rho$ and decrease in the case of small and negative $\rho$. Contrary, the dependence of the size of $\sigma_{\text {tot }}$ on the coefficient $C$ is linear. From Tables 2-5 it is clear that the negative size of $C$ can be only with large negative $\rho$. The minimum $\sum_{i=1}^{N} \chi_{i}^{2}$ was obtained with large positive $C$. Again, we can see that the inclusion of the Coulomb dependence terms leads to an increase of the size of $\sigma_{\text {tot }}$.

In our opinion, this analysis shows the problems when we try to measure $\sigma_{t o t}(s)$ and $\rho(s, t)$ in the different experiments. Hence, to avoid such a situation, we need to determine $\sigma_{\text {tot }}(s)$ and $\rho(s, t)$ simultaneously in one experiment.

\section{Regge representation}

In the previous analysis the simplest phenomenological model with many assumptions (for example, the equality of the slopes of the imaginary and real parts and others) was used. Now let us take also the simple model but which is based on the Regge representation for the scattering amplitude and was used in the Donnachi-Landshoff [16] model. In this case, the real part is not fitted but is determined by the form of the scattering amplitude. We take the hadron form-factor in the standard electromagnetic form. Hence, some part of the $t$ dependence of the scattering amplitude is fixed.

The hadron spin non-flip amplitude was chosen in the form

$$
F(s, t)=i h \hat{s}^{\Delta} f_{1}(t)^{2} \frac{\sigma_{t o t}}{4 \pi 0.38938} e^{\left[\alpha_{1}^{\prime} t+\alpha_{2}^{\prime}\left(\sqrt{4 \mu^{2}-t}-2 \mu\right)\right] \operatorname{Ln}(\hat{s})}
$$

with the electromagnetic form factor $f_{1}(t)(\underline{6})$ and $\hat{s}=s e^{-i * \pi / 2} ; \mu$ is the pion mass. We take $h \operatorname{Re}\left(\hat{s}^{\Delta}\right)=1$ at $\sqrt{s}=7 \mathrm{TeV}$.

We examined two sizes of the intercept $\alpha(0)=1+\Delta$ with a) $\Delta=0.1$ (Table 8 ) and b) $\Delta=0.08$ (Table 9 ). These sizes of the intercept lead to $\rho(0)=0.156$ and $\rho(0)=0.128$ at $\sqrt{s}=7 \mathrm{TeV}$, respectively. The $\sum_{i=1}^{N} \chi_{i}^{2}$ is slightly above the minimal $\sum_{i=1}^{N} \chi_{i}^{2}$ in the pure phenomenological cases if we do not fix $\sigma_{\text {tot }}$. Contrary, if we take the size of $\sigma_{t o t}=98.4 \mathrm{mb}$, as obtained 


\begin{tabular}{|c|c|c|c|c|}
\hline & $\Delta=0.1$ & \multicolumn{3}{|c|}{$\rho(\sqrt{s}=7 \mathrm{TeV}, t=0)=0.156$} \\
\hline$N$ & $\sum_{i=1}^{N} \chi_{i}^{2}$ & $\alpha_{1}^{\prime}$ & $\alpha_{2}^{\prime}$ & $\sigma_{t o t}, m b$ \\
\hline 47 & 65.2 & 0.325 & 0.fix & $99.7 \pm 0.15$ \\
\hline 47 & 65.1 & 0.328 & $-0.002 \pm 0.015$ & $99.6 \pm 0.2$ \\
\hline 40 & 58.1 & 0.324 & $0 . f i x$ & $99.6 \pm 0.05$ \\
\hline 40 & 55.8 & 0.276 & $0.037 \pm 0.02$ & $99.9 \pm 0.26$ \\
\hline 47 & 204 & 0.314 & 0.fix & $98.4 f i x$ \\
\hline 47 & 95.3 & 0.427 & $-0.075 \pm 0.008$ & $98.4 f i x$ \\
\hline 40 & 154 & 0.312 & $0 . f i x$ & $98.4 f i x$ \\
\hline 40 & 90.1 & 0.437 & $-0.08 \pm 0.01$ & $98.4 f i x$ \\
\hline
\end{tabular}

Table 8: The basic parameters of the Regge amplitude are determined by fitting experimental data with free $\sigma_{t o t}$.

by the TOTEM Collaboration, $\chi^{2}$ increases essentially, especially we do not include in the fitting procedure the additional slope $\alpha_{2}^{\prime}$. This coefficient is determined if we examine the short interval of momentum transfer with the number of experimental points 40 .

Comparing the results in Tables 8 and 9 we see that $\sum_{i=1}^{N} \chi_{i}^{2}$ is slightly less in the second case. However, the obtained sizes of the total cross sections are practically the same. Hence, the taken interval of the intercept and correspondingly $\rho$ lead to the small difference in the fitting sizes of $\sigma_{\text {tot }}$. Such representation shows that the slope has some complicated form and it is necessary to be taken into account in the pure phenomenological analysis.

Comparing the results of the Regge approaches with the results of the pure phenomenological model we see that the maximal $\sigma_{t o t}$ are the same, near $100.9 \mathrm{mb}$, but the minimal sizes are less in the case of the pure phenomenological model, near $97.18 \mathrm{mb}$. In last case the size of $\rho$ is near zero. Hence the value of $\rho \sim 0.1 \div 0.15$ leads a larger $\sigma_{\text {tot }}$ than obtained by the TOTEM Collaboration.

\section{The size of $\rho$}

Let us check up our assumptions about the size and momentum transfer dependence of the real part of the scattering amplitude. We can use the 


\begin{tabular}{c|c|c|c|c}
\hline \multicolumn{4}{c}{$\Delta=0.08$} & \multicolumn{3}{c}{$\rho(\sqrt{s}=7 \mathrm{TeV}, t=0)=0.128$} \\
\hline \hline$N$ & $\sum_{i=1}^{N} \chi_{i}^{2}$ & $\alpha_{1}^{\prime}$ & $\alpha_{2}^{\prime}$ & $\sigma_{\text {tot }}, \mathrm{mb}$ \\
\hline & & & & \\
47 & 64.4 & 0.325 & $0 . f i x$ & $100.0 \pm 0.1$ \\
47 & 64.3 & 0.338 & $0.008 \pm 0.003$ & $99.9 \pm 0.1$ \\
40 & 56.4 & 0.323 & $0 . f i x$ & $99.9 \pm 0.12$ \\
40 & 55.3 & 0.299 & $0.005 \pm 0.004$ & $100.2 \pm 0.3$ \\
47 & 285 & 0.310 & $0 . f i x$ & 98.4 fix \\
47 & 106.7 & 0.455 & $-0.096 \pm 0.008$ & 98.4 fix \\
40 & 211 & 0.307 & $0 . f i x$ & 98.4 fix \\
40 & 99.6 & 0.473 & $-0.108 \pm 0.011$ & 98.4 fix \\
\hline
\end{tabular}

Table 9: The basic parameters of the model are determined by fitting experimental data with free $\sigma_{t o t}$.

method which was proposed and explored in [17, 18, 19, 20], and introduce the value

$$
\Delta_{R}^{t h}(s, t)=\left(R e F_{C}(t)+\operatorname{Re}_{h}(s, t)\right)^{2} \geq 0
$$

From the eq.(9)

$$
\Delta_{R}^{e x p}\left(s, t_{i}\right)=\left[\left.\frac{d \sigma}{d t_{i}}\right|_{\text {exp. }} / n-k \pi *\left(I m F_{c}\left(t_{i}\right)+\operatorname{Im} F_{h}\left(t_{i}\right)\right)^{2}\right] /(k \pi) .
$$

where $n$ is the additional normalization coefficient which is reflect the errors in the Luminosity determination and other possible systematical errors. For the proton-proton high energy scattering the real part of the hadron scattering amplitude is positive at small momentum transfer, and Coulomb amplitude is negative and exceeds the size of the hadronic part of the amplitude at $t \rightarrow 0$, but has a large slope. Hence, $\Delta_{R}(t)$ will have the minimum at some value $t$ and then a wide maximum. Comparing $\Delta_{R}^{t h}(s, t)$ with $\Delta_{R}^{e x p}\left(s, t_{i}\right)$ gives the accuracy of the experiment and of the theoretical model assumptions.

Let us take the parameters obtained the TOTEM Collaboration $\sigma_{\text {tot }}=$ $98.6 \mathrm{mb}, B=19.9 \mathrm{GeV}^{-2}, n=1, \rho(0)=0.141$ and calculate the value $\Delta_{R}^{t h}(s, t)$, eq. (13) . The result is shown in Fig.3 by the hard line. Now let us take these parameters for the imaginary part of the scattering amplitude and calculate $\Delta_{R}^{e x p}\left(s, t_{i}\right)$ using eq.(14). The triangles in Fig.3 present these 
calculations. Obviously, the first and second calculations are very far from each other. If we take the real part with the parameters $\sigma_{t o t}=96.4 \mathrm{mb}$, $B=19.9 \mathrm{GeV}^{-2}, \rho=0.1$ and calculate $\Delta_{R}^{t h}(s, t)$ (short dashed line in Fig.3) the position of the minimum moves to higher $t$, but the difference remains large.

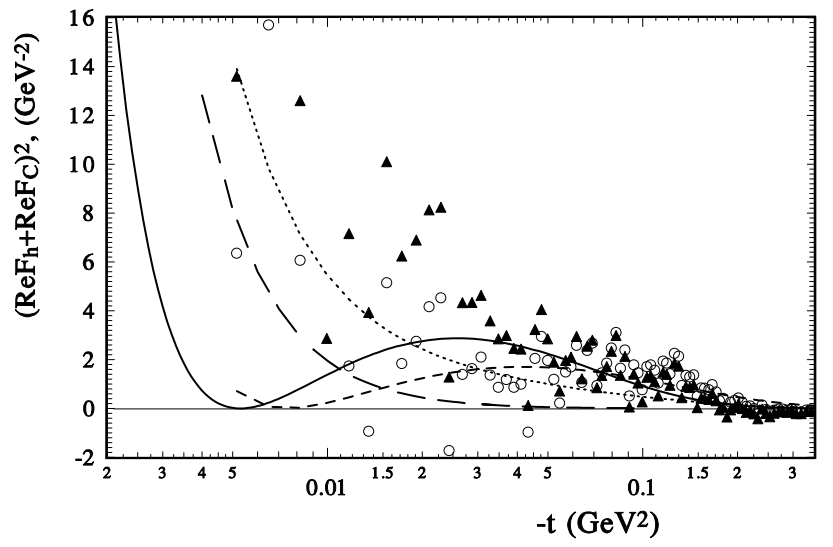

Figure 3: $\Delta_{R}^{e x p}\left(t_{i}\right)$ eq.(14): triangles - calculations with the TOTEM parameters; circles - calculations with $\sigma_{t o t}=96.4 \mathrm{mb}, B=20.3 \mathrm{GeV}^{-2}, n=1.08, C=-0.05$; hard line eq.(13) the real part with TOTEM parameters and $\rho=0.14$; short dashed line $-\Delta_{R}^{t h}(s, t)$ eq.(13) with $\sigma_{\text {tot }}=96.4 \mathrm{mb}, B=19.9 \mathrm{GeV}^{-2}$ and $\rho=0.1$; long dashed line - eq.(13) with $\rho=0$; points line - eq.(13) with $\sigma_{\text {tot }}=96.4 \mathrm{mb}, B=19.9 \mathrm{GeV}^{-2}$ and $\rho=-0.05$.

If we take some other parameters for the imaginary part $\sigma_{t o t}=96.4 \mathrm{mb}$, $B=20.3 \mathrm{GeV}^{-2}, n=1.08, C=-0.05 \mathrm{GeV}^{-1}$, we obtain from eq. (14) the results for $\Delta_{R}^{e x p}\left(t_{i}\right)$, which is shown in Fig.3 by circles. The $\Delta_{R}^{t h}(s, t)$ with only the Coulomb amplitude $(\rho=0)$ is shown in Fig.3 by the long dashed line, and the dotted line presents the calculation with $\rho=-0.05$. We can see that only in the last case the difference between the calculations by eqs.(14) and (13) is not large. That is why our fitting procedure in most part requires the size of $\rho$ near zero or negative. But these parameters for the imaginary and real parts of the scattering amplitude are far from the parameters obtained by the TOTEM Collaboration. This situation is unclean. Maybe, there is some problem with the normalization of the separate parts of the experimental data, or there exists some additional (probably oscillation) term (see [21]) which changes the form of the imaginary part. 


\section{Conclusion}

The analysis of the new experimental data obtained by the LHC TOTEM Collaboration [2, 4] shows that there are some additional specific moments which are to be taken into account in determining the size of the total cross sections. We found that it is necessary to take into account the electromagnetic interactions in the analysis of the experimental data. In this case, the impact of the size of $\rho$ on the determination of $\sigma_{\text {tot }}$ increases in comparison

with the standard factor $1 / \sqrt{1+\rho^{2}}$. The deviation of the hadron part of the scattering amplitude at small momentum transfer from the standard exponential form can be taken into account by the additional part of the slope proportional to $q$. This impact will increase when the new experimental data will be obtained for smaller $t$. It is needed to check out the obtained, during the fitting procedure, real part of the hadron amplitude by using eq.(14). Now our calculations show the inconsistency of the size of $\rho=0.14$ with the parameters of the scattering amplitude obtained by the TOTEM Collaboration. Maybe, it is necessary to explore in more detail the non-exponential behavior of the real and imaginary part s and a possibility of the presence of some oscillation term.

Finally, we should note that the best way to decrease the impact of the different assumptions, which are examined in the phenomenological model, consists in the determination of the sizes of $\sigma_{t o t}$ and $\rho(s, t)$ simultaneously in one experiment.

\section{References}

[1] G. Anelli, et al., JINST 3 (2008) S08007.

[2] G. Latino et all. (TOTEM Coll.), arXiv: 1110.1008.

[3] J. R. Cudell, V. Ezhela, K. Kang, S. Lugovsky and N. Tkachenko, Phys. Rev. D 61 (2000) 034019 [Erratum-ibid. D 63 (2001) 059901] arXiv:hep-ph/9908218]; J. R. Cudell et al., Phys. Rev. D 65 (2002) 074024 [arXiv:hep-ph/0107219].

[4] The TOTEM Collaboration (G. Antchev et al.) EPL, 95 (2011)41001.

[5] R. Fiore, L. Jenkovszky, R. Orava, E. Predazzi, A. Prokudin, O. Selyugin, Mod.Phys., A24: 2551-2559 (2009) 
[6] J. R. Cudell and O. V. Selyugin, Czech. J. Phys. 54 (2004) A441 arXiv:hep-ph/0309194.

[7] J. R. Cudell and O. V. Selyugin, Phys. Rev. Lett. 102 (2009) 032003 arXiv:0812.1892 [hep-ph]].

[8] O. V. Selyugin, Sov. J. Nucl. Phys. 55, 466 (1992) [Yad. Fiz. 55, 841 (1992)].

[9] O. Selyugin, Phys. Lett. B333,(1994) 245.

[10] O.V. Selyugin, Mod. Phys. Lett. A9 (1994) 1207.

[11] O.V. Selyugin, Mod. Phys. Lett. A14, 223 (1999).

[12] O.V. Selyugin, Phys. Rev. D 60 (1999) 074028.

[13] N. H. Buttimore, E. Gotsman, E. Leader, Phys. Rev. D 35, 407 (1987).

[14] A.A. Anselm and V.N. Gribov, Phys.Lett. B40, 487 (1972).

[15] D.A. Fagundes, et all, hep-ph[1306.0452]

[16] A. Donnachi, P.V. Landshoff, Phys.Lett. B387 (1996) 637.

[17] O.V. Selyugin, Proceedings VI Intern. Conf. On Diffraction and Elastic scattering", Blois, France,(1995).

[18] O.V. Selyugin, Talk on X-th Intern. Conf. On Diffraction and Elastic scattering", Xelsinki, Finland,(2003), arXiv.org: hep-ph/0306256.

[19] O.V. Selyugin, arXiv.org: hep-ph/0104295].

[20] P. Gauron, B. Nicolescu, O.V. Selyugin, Phys.Lett. B629 (2005) 83.

[21] O. V. Selyugin, J.-R. Cudell International workshop " Diffraction-10", Italy, September 10 - 15, Otranto (Lecce), Italy (2010); arXiv:1011.4177. 